\title{
Anti-zinc transporter protein 8 autoantibodies significantly improve the diagnostic approach to type 1 diabetes: an Italian multicentre study on paediatric patients
}

\author{
Martina Fabris $^{1,2} \cdot$ Silvia Zago $^{2} \cdot$ Marco Liguori $^{3} \cdot$ Maria Teresa Trevisan $^{4}$. \\ Manuela Zanatta ${ }^{5}$ Alberto Comici ${ }^{6}$ - Giorgio Zanette ${ }^{7} \cdot$ Eva Carlin $^{8}$. \\ ${\text { Francesco } \text { Curcio }^{1,2} \cdot \text { Elio Tonutti }}^{9}$
}

Received: 13 December 2014/ Accepted: 26 June 2015/Published online: 21 July 2015

(C) The Author(s) 2015

\begin{abstract}
Background and aim Anti-ZnT8 antibodies (ZnT8A) were recently proposed as a new independent serological marker in Type 1 diabetes (T1D), leading to a significant improvement of the positive predictive value of autoantibody measurement in this setting. The aim of this retrospective multicentre study was to investigate ZnT8A as a complement to the current T1D autoantibody assays in a large cohort of paediatric Italian patients.

Methods ZnT8A were assessed by ELISA in 213 T1DM paediatric patients referred to six different centres in North-
\end{abstract}

Electronic supplementary material The online version of this article (doi:10.1007/s13317-015-0068-4) contains supplementary material, which is available to authorized users.

Martina Fabris

fabris.martina@aoud.sanita.fvg.it

1 Institute of Clinical Pathology, University Hospital of Udine, Piazzale S. Maria Misericordia 15, 33100 Udine, Italy

2 Department of Medical and Biological Sciences, University of Udine, Udine, Italy

3 Laboratory of Clinical Pathology, Brotzu Hospital, Cagliari, Italy

4 Unit of Laboratory Medicine, G. Fracastoro Hospital, San Bonifacio, Verona, Italy

5 Clinic of Pediatrics, University Hospital of Udine, Udine, Italy

6 Unit of Pediatrics, San Daniele del Friuli Hospital, Udine, Italy

7 Unit of Diabetology, Santa Maria degli Angeli Hospital, Pordenone, Italy

8 Unit of Pediatrics, Latisana Hospital, Udine, Italy

9 Laboratory of Immune Pathology and Allergy, University Hospital of Udine, Udine, Italy
East Italy. Fifty-four were analysed at disease onset, 79 within 4 years from diagnosis and 80 after 5 or more years from diagnosis. Retrospective data about islet cell autoantibodies (ICA), anti-insulin (IAA), anti-glutamate decarboxylase (GADA) and anti-protein tyrosine phosphatase IA-2 (IA-2A) antibodies were collected and compared.

Results Overall, ZnT8A showed positive results in 106/213 (49.8\%) T1D patients and were found in 10 $(4.7 \%)$ subjects previously classified as autoantibody negative based on the existing markers (GADA, IA-2A, IAA and ICA), increasing the overall diagnostic sensitivity from 85.9 to $90.6 \%$. ZnT8A disclosed the same sensitivity $(61.1 \%)$ at disease onset as GADA $(61.1 \%)$ and higher than IA-2A (53.7\%), with only GADA showing much persistence in the long-term follow-up. Focusing on patients at disease onset, all the ICA positive were associated with at least one positive autoantibody among GADA, IA-2A and ZnT8A, $16.7 \%$ of whom presenting only anti-ZnT8-positive antibodies.

Conclusion This study confirms ZnT8A as an important additional and independent diagnostic marker of T1D and supports its introduction in the routine diagnostic process to replace less sensitive methods and improve the overall autoantibody sensitivity.

Keywords Autoimmune diabetes - Autoantibodies · Anti-ZnT8 antibodies · Paediatrics · Sensitivity

\section{Introduction}

Type 1 diabetes (T1D) is a complex, multifactorial disease caused by the selective destruction of the insulin-producing pancreatic $\beta$ cells [1]. The appearance 
of cellular and humoral islet cells autoimmunity is the hallmark of the disease process, and a number of $\beta$ cell proteins are known to be targets of autoreactive B and $\mathrm{T}$ cells. Identification of these molecular targets provided pivotal insight into the pathogenic process, diagnostic assays and potential therapeutic strategies [2].

The best-known serological markers of T1D are autoantibodies to islet cell antigens (ICA) [3], such as antiinsulin (IAA) [4], anti-glutamate decarboxylase (GADA) [5] and anti-protein tyrosine phosphatase (PTP)-like antigen IA-2 (IA-2A) [6] antibodies. These biomarkers are evidence of the autoimmune process that precedes the clinical onset of T1D and the histological determination of ICA or the combined measurement of IAA, GADA and IA$2 \mathrm{~A}$ can identify $80 \%$ or more of patients at disease onset or at risk of developing disease [7].

Recently, a new, high-ranking diabetes autoantigen was identified in the zinc transporter protein 8 (ZnT8 or SCL30-A8) [8, 9]. Zinc transporter (ZnT) proteins of the SCL30 (solute carrier family 30) gene family regulate zinc efflux from the cytoplasm to the extracellular matrix or into intracellular vesicles. ZnT8, a polytopic integral membrane protein, is mainly expressed in the endocrine pancreatic tissue and is responsible for the very high level of zinc accumulation in the insulin secretory granules [10]. The gene encoding for ZnT8 maps to chromosome $8 \mathrm{q} 24.11$ and presents a polymorphism at position 325 (Arg325Trp) [11], which could influence also the development of ZnT8 immunogenicity and the phenotypic features of paediatric T1D [12, 13] and adultonset type 2 diabetes [14], indicating that this amino acid substitution has the critical role in antibody binding [15]. The ZnT8 represent a major player to provide zinc for insulin maturation and/or storage processes in insulinsecreting pancreatic $\beta$-cells [10]. Therefore, antibodies against ZnT8 may affect several important processes, such as insulin synthesis, storage, secretion, and may impair islet cell paracrine/autocrine communication as well [10].

The combined measurement of ZnT8A, GADA, IA$2 \mathrm{~A}$ and IAA raised diabetes autoimmunity detection rates to $98 \%$ at disease onset, a level that approaches that needed to detect pre-diabetes in general paediatric population [9]. Furthermore, ZnT8A and IA-2A were recently associated with childhood-onset and acute-onset patients [16].

The aim of this multicentre study was to evaluate the diagnostic impact of ZnT8A investigation at disease onset and in the follow-up, as a complement to the common diabetes autoantibodies in a large cohort of "real-life" paediatric diabetic patients referred to North-East Italy.

\section{Methods}

This retrospective study was conducted in 213 T1D paediatric patients (45.7\% females and $54.3 \%$ males, mean age $11.5 \pm 4.2$ years): 54 at disease onset, 79 within $1-4$ years after diagnosis and 80 at $\geq 5$ years from diagnosis. Patients referred to six different centres in NorthEast Italy and were compared with 110 age and sex-matched controls $(48.2 \%$ females and $51.8 \%$ males, mean age $9.6 \pm 4.9$ years) recruited at the Clinic of Pediatrics in Udine, among ambulatory patients. Informed consent was obtained from all individuals included in the study. ZnT8A were analysed in patients and controls by enzyme-linked immunosorbent assays (ELISA; Dia.Metra Srl, Milan, Italy) in serum aliquots obtained from blood samples required for other necessary laboratory analyses and stored at $-80{ }^{\circ} \mathrm{C}$ until analysis. As regards the control population, diabetes and/or family history of diabetes, and the most frequently diabetes-associated autoimmune disease (i.e. coeliac disease, Hashimoto's thyroiditis) were excluded. GADA and IA-2A were analysed by diagnostic ELISA methods in all patients. IAA were analysed by ELISA (Orgentec Diagnostik, Mainz, Germany) in 131 cases and retrospective data about ICA investigated by indirect immunofluorescence (IFI) test on cryostat primate's pancreas (IMMCO, Buffalo, NY) were available in 130 cases. Statistic analyses were performed using the Graph Pad Prism and Instat softwares (San Diego CA), considering significant differences when $p<0.05$.

\section{Results}

Considering the cut-off suggested by the manufacturer ( $>15 \mathrm{AU} / \mathrm{ml}$ ), positive ZnT8A were found in 106/213 $(49.8 \%)$ T1D patients versus 3/110 (2.7\%) controls (Mann-Whitney's $t$ test, $p<0.0001$, Fig. 1). At the cut-off $>15 \mathrm{AU} / \mathrm{ml}$, the ROC curve plotting our patient versus control data showed a sensitivity of $97.3 \%$ and a specificity of $50.7 \%$ (see supplementary data). All the three positive control sera disclosed clear-cut positive ZnT8A, in two of them (respectively, 178 and $215 \mathrm{AU} / \mathrm{ml}$ ), all the other autoantibodies were negative, while the one with the highest ZnT8A concentration $(1554 \mathrm{AU} / \mathrm{ml})$ presented also high positive GADA and IA-2A, suggesting to be a child at high risk to develop diabetes in the next 5 years. In all the other control sera, ZnT8A resulted undetectable. As indicated by the ROC curve, when lowering the cut-off to $>10 \mathrm{AU} / \mathrm{ml}$, we increased the overall sensitivity up to $60 \%$, without loosing specificity. Among these 22 cases with ZnT8A between 10 and $15 \mathrm{AU} / \mathrm{ml}$, only three were not associated with other autoantibodies. No patients showed detectable 
Fig. 1 ZnT8A in T1D patients and control subjects. Overall, patients showed positive antiZnT8 autoantibodies in $49.7 \%$ $(106 / 213)$ versus $2.7 \%(3 / 110)$ positive controls

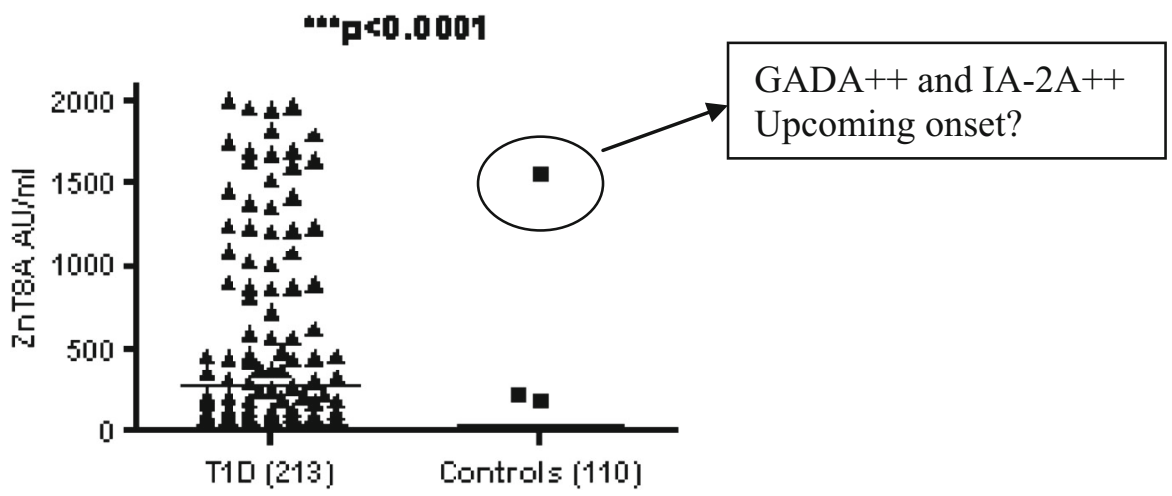

ZnT8A below $10 \mathrm{AU} / \mathrm{ml}$. ZnT8A prevalence tended to be higher in younger patients, accounting for $56.3 \%(18 / 32)$ among $0-6$ years subgroup, $48.7 \%$ (37/76) among $7-12$ years subgroup and $48.6 \%$ (51/105) among 13-18 years subgroup, but the differences were not statistically significant.

The sensitivity of ZnT8A at disease onset was $61.1 \%$ (similar to that showed for GADA) and remained substantially stable in patients analysed after one up to 4 years from diagnosis (56 and $59.3 \%$ ), then dropped down significantly in patients $\geq 5$ years from diagnosis ( $33.8 \%$; Fig. 2a). IA-2A showed a very similar behaviour (Fig. 2b), while GADA showed higher sensitivity in patients within 1 year from diagnosis and longer persistence after $\geq 5$ years from diagnosis (Fig. 2c).

Data about IAA analysed by ELISA were available in 131 cases. Overall, 41/131 (32.1\%) disclosed positive results. In the subgroup at onset (29 patients), only two $(6.9 \%)$ presented low-positive IAA. Since the ELISA for IAA detection showed this extremely low sensitivity at disease onset, and was not able to distinguish antibodies against exogenous and endogenous insulin in the followup, comparisons and correlations between ZnT8A and IAA were not taken into major consideration in this study.

A disease onset, $6 / 54(11.5 \%)$ patients presented only ZnT8A-positive antibodies (negative GADA, IA-2A and IAA), only two of them associated with positive ICA. Thus, thanks to ZnT8A assessment, at disease onset, autoantibody-positive patients increased from $40 / 54$ $(74.1 \%)$ to $46 / 54(85.2 \%)$. In the remaining cases ( $\geq 1$ year from diagnosis), we found another four ZnT8A isolated positive patients (only one with available data about ICA IFI test, that was negative), increasing the overall diagnostic sensitivity of the laboratory investigations from 85.9 to $90.6 \%$.

We finally analysed separately the 130 patients with available retrospective data of ICA IFI investigation on primate's pancreas. In total, 46 (35.4\%) were reported as
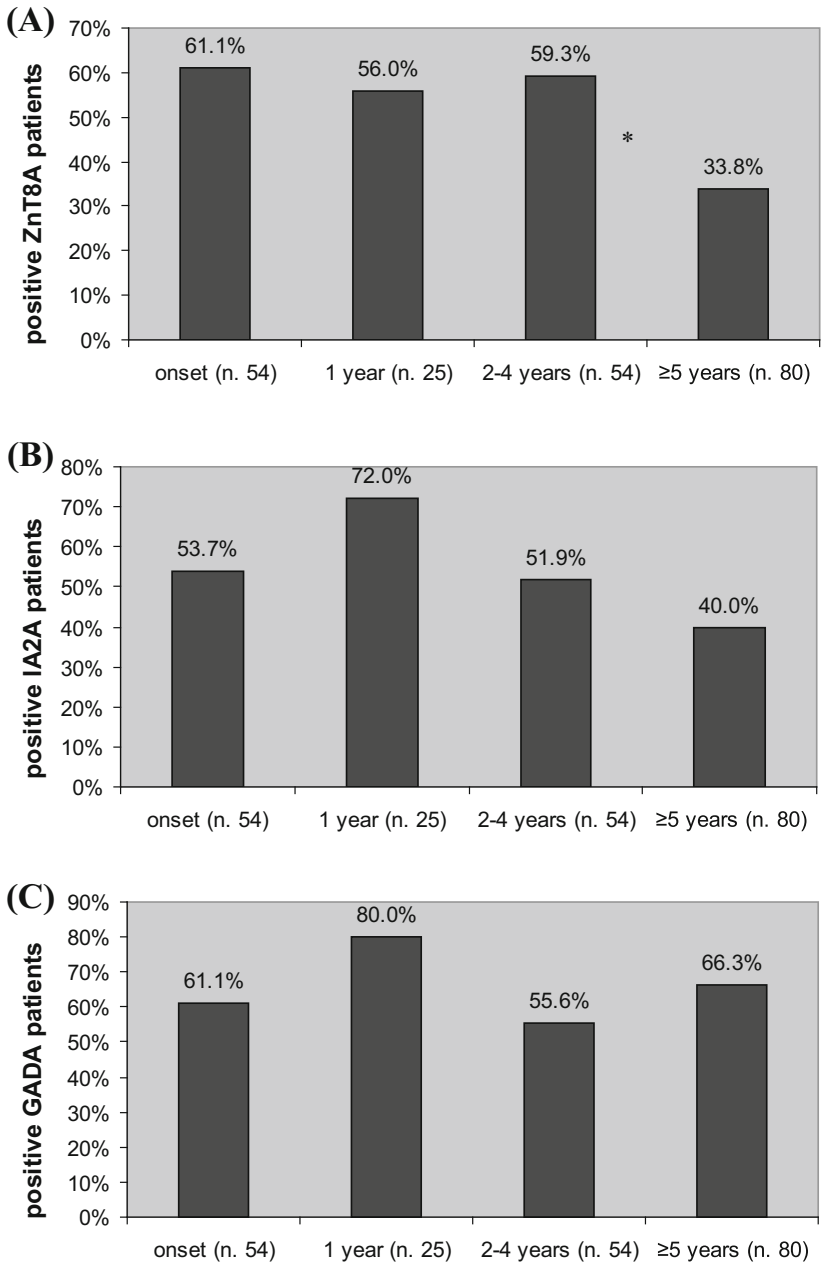

Fig. 2 Persistence of positive autoantibodies in the follow-up after diagnosis. a The percentage of positive ZnT8A patients remained substantially stable up to 4 years from diagnosis, then decreased significantly in patients analysed $\geq 5$ years from diagnosis $(79 / 133$, $59.4 \%$ within 4 years versus $27 / 80,33.8 \%$ in $\geq 5$ years patients; $* p=0.0004,95 \%$ CI 1.61-5.12, OR 2.87). b Similarly, IA-2A positivity picked 1 year from diagnosis and dropped significantly $\geq 5$ years from diagnosis). c The prevalence of positive GADA was very high 1 year from diagnosis, then slightly decreased remaining as high as at disease onset in patients analysed $\geq 5$ years from diagnosis 
Table 1 Prevalence of specific autoantibodies in ICA-positive (ICA+) and ICA-negative (ICA-) patients

\begin{tabular}{llllllc}
\hline & No. & GADA+ $(\%)$ & IA-2A+ $(\%)$ & ZnT8A+ $(\%)$ & autoAbs negative & Only ZnT8A+ \\
\hline All patients (no. 130) & & & & & $4(8.7 \%)$ & $4(8.7 \%)$ \\
$\quad$ ICA+ & 46 & 52.2 & $58.7 *$ & 43.5 & $18(21.4 \%)$ & $5(5.9 \%)$ \\
ICA- & 84 & 60.7 & $35.7 *$ & 47.6 & & \\
At onset patients (no. 35) & & & & & $0(0 \%)$ & $2(16.7 \%)$ \\
ICA+ & 12 & 50 & 66.7 & 83.3 & $7(30.4 \%)$ & $3(13 \%)$ \\
ICA- & 23 & 52.2 & 39.1 & 52.2 & & \\
\end{tabular}

In the overall series of patients with available data about ICA IFI investigation, GADA- and ZnT8A-positive patients did not differed significantly in ICA+ and ICA - subjects, while IA-2A were more prevalent in ICA+ (*OR 2.56, $95 \%$ CI 1.22-5.35, $p=0.016)$. Of note, only 4/130 (3.1\%) were positive only at ICA investigation versus 5/130 (3.8\%) positive only at ZnT8A investigation. When considering only patients at disease onset, ICA+ cases were completely intercepted by at least one positive autoantibody among GADA, ZnT8A and IA-2A. Furthermore, the ZnT8A contribution allowed to detect 3/35 (8.6\%) of patients who previously were classified as all negative (ICA and specific autoantibodies)

positive. In contrast, $75(57.7 \%)$ were GADA positive, 57 (43.8\%) IA-2A positive and $60(46.2 \%)$ ZnT8A positive. Thus, the global sensitivity of ICA was much lower than those of the individual autoantibodies. Furthermore, as illustrated in Table 1, a significant percentage (66/84, $78.6 \%$ ) of patients classified as ICA negative were positive for at least one of the other autoimmune biomarkers, while only four patients $(4 / 46,8.7 \%)$ were found to be autoantibody negative among the ICA-positive cases. Of note, when focusing on the 35 patients analysed at disease onset, all $(100 \%)$ the 12 ICA-positive patients were associated with at least one positive autoantibody among GADA, IA-2A and ZnT8A, and among the 23 ICA-negative patients, seven $(30.4 \%)$ recognised at least one positive autoantibody, that was ZnT8A in three cases (Table 1). Thus, the positive predictive value of the combination of all the specific autoantibody investigations, especially when including also ZnT8A, appeared much higher than that of the ICA IFI test.

\section{Discussion}

This large Italian multicentre retrospective study confirmed the important improvement associated to the introduction of the ZnT8A detection in the real-life laboratory diagnostic process of paediatric T1D. The sensitivity at disease onset $(61.1 \%)$ and the specificity $(97.3 \%)$ of the ZnT8A assay in our series of paediatric T1D were in line with previous studies [9, 17, 18] and comparable with those of the other major known diabetes autoantibodies, confirming the analytical robustness and the clinical usefulness of this test and supporting its widespread integration in other Italian laboratories. When setting the cut-off slightly lower than suggested by the manufacturer (i.e. $<10 \mathrm{AU} / \mathrm{ml}$ instead of $<15 \mathrm{AU} / \mathrm{ml}$ ), the overall sensitivity of the test increased significantly, without loosing any specificity. Therefore, ZnT8A values between 10 and $15 \mathrm{AU} / \mathrm{ml}$ deserve to be mentioned as "borderline" in the medical report, eventually suggesting to repeat the analysis. Concerning the positive control sera, the one associated with GADA and IA-2A may be surely considered at high risk for T1D development in the near future, but also the others, showing lower, but clear-cut positive ZnT8A, deserve to be closely followed for a possible future development of diabetes.

Human ZnT8 is specifically expressed not only in the pancreatic $\beta$-cells, but also in the follicle epithelial cells and in the parafollicular cells of the thyroid gland [19], and, very recently, anti-ZnT8 antibodies cross-reacting with mycobacterium avium paratuberculosis were found to be highly common in Hashimoto's Thyroiditis (HT) from Sardinia [20]. So that, positive ZnT8A controls should also be screened for HT development.

In agreement with another Caucasian series of T1D children [17], the prevalence of ZnT8A remained substantially stable in patients up to four years from the clinical diagnosis, but then showed a significant decrease, and the ZnT8A remained finally appreciable in approximately $34 \%$ of patients evaluated $\geq 5$ years from diagnosis. Thus, ZnT8A, as assessed by this commercial ELISA, disclosed the same sensitivity at disease onset as GADA and slightly higher than IA-2A, with only GADA showing much persistence in the very long follow-up.

In our series, the prevalence of ZnT8A tended to be higher in young children versus teenagers, but the difference was not statistically significant. The relationship between ZnT8A and age is actually controversial since in Chinese children, ZnT8A prevalence was lower in older patients [21], while in a very large Finnish study ZnT8A positivity was associated with older age at diagnosis [22].

Anyway, we definitely confirmed ZnT8A as a reliable marker of diabetes autoimmunity, either independently, in a significant fraction of previously defined autoantibodynegative subjects, or in conjunction with other autoantibodies, increasing the overall diagnostic performance as well as the positive predictive value of the laboratory 
investigations. The assessment of ZnT8A as a complement of the current T1D bioanalysis appears of added importance when considering that IAA by ELISA and ICA IFI test, at least when performed by the methods that are currently available in our laboratory, presented several technical limitations and did not show a valuable impact on the diagnostic process. IAA by radioimmunometric methods can only be performed in a reference diabetic centre, due to the high cost and the specific needs of this method [23]. Similarly, ICA by IFI on primate's tissue, that is not the gold standard method for ICA investigation, but that is hardly assessable on human tissue in real practice, presented, in our series, a much lower sensitivity if compared to the individual antibodies and more to the combination of all the specific autoantibodies.

Of note, in the present series, when adding ZnT8A investigation, IAA and ICA never represented the sole autoantibody positivity at disease onset. Thus, when introducing ZnT8A as a complement of the current biomarkers, the overall diagnostic performance of the serological tests increased significantly, possibly rendering IAA and ICA excludable without loosing sensitivity.

Therefore, considering the low sensitivity, the subjectivity, the cost and the limitations of ICA IFI investigation on primate's pancreas, ZnT8A may represent a valid replacement of this assay at disease onset in paediatric patients. Of note, ZnT8A were even proposed as IA-2A replacement in Indian T1D patients, without loss of sensitivity and specificity [18].

In conclusion, ZnT8A investigation is currently performed only in a limited number of Italian laboratories, but our present data support a wider diffusion and lead us to propose the combination of GADA, IA-2A and ZnT8A as the most powerful and cost-effective diagnostic approach in paediatric T1D patients at this moment.

\section{Compliance with Ethical Standards}

Conflict of interest All authors declare that they have no conflict of interest.

Informed consent Informed consent was obtained from all individual participants included in the study.

Ethical approval All procedures performed in this study were in accordance with the ethical standards of the institutional research committee and with the 1964 Helsinki declaration and its later amendments or comparable ethical standards. For this type of study, formal consent is not required.

\section{References}

1. Gillespie KM (2006) Type 1 diabetes: pathogenesis and prevention. Can Med Assoc J 175:165-170
2. Vives-Pi M, Rodríguez-Fernández S, Pujol-Autonell I (2015) How apoptotic $\beta$-cells direct immune response to tolerance or to autoimmune diabetes: a review. Apoptosis 20(3):263-272

3. Bottazzo GF, Florin-Christensen A, Doniach D (1974) Islet-cell antibodies in diabetes mellitus with autoimmune polyendocrine deficiencies. Lancet 2:1279-1283

4. Palmer JP, Asplin CM, Clemons P, Lyen K, Tatpati O, Raghu PK, Paquette TL (1983) Insulin antibodies in insulin-dependent diabetics before insulin treatment. Science 222:1337-1339

5. Baekkeskov S, Aanstoot HJ, Christgau S, Reetz A, Solimena M, Cascalho M, Folli F, Richter-Olesen H, De Camilli P (1990) Identification of the $64 \mathrm{~K}$ autoantigen in insulin-dependent diabetes as the GABA-synthesizing enzyme glutamic acid decarboxylase. Nature 347:151-156

6. Bonifacio E, Lampasona V, Genovese S, Ferrari M, Bosi E (1995) Identification of protein tyrosine phosphatase-like IA2 (islet cell antigen 512) as the insulin-dependent diabetes-related $37 / 40 \mathrm{~K}$ autoantigen and a target of islet-cell antibodies. J Immunol 155:5419-5426

7. Achenbach P, Warncke K, Reiter J, Naserke HE, Williams AJ, Bingley PJ, Bonifacio E, Ziegler AG (2004) Stratification of type 1 diabetes risk on the basis of islet autoantibody characteristics. Diabetes 53:384-392

8. Wenzlau JM, Juhl K, Yu L, Moua O, Sarkar SA, Gottlieb P, Rewers M, Eisenbarth GS, Jensen J, Davidson HW, Hutton JC (2007) The cation efflux transporter ZnT8 (Slc30A8) is a major autoantigen in human type 1 diabetes. Proc Natl Acad Sci USA 104:17040-17045

9. Wenzlau JM, Moua O, Sarkar SA, Yu L, Rewers M, Eisenbarth GS, Davidson HW, Hutton JC (2008) SIC30A8 is a major target of humoral autoimmunity in type 1 diabetes and a predictive marker in prediabetes. Ann NY Acad Sci 1150:256-259

10. Davidson HW, Wenzlau JM, O'Brien RM (2014) Zinc transporter $8(\mathrm{ZnT} 8)$ and $\beta$ cell function. Trends Endocrinol Metab 25(8):415-424

11. Wenzlau JM, Liu Y, Yu L, Moua O, Fowler KT, Rangasamy S, Walters J, Eisenbarth GS, Davidson HW, Hutton JC (2008) A common nonsynonymous single nucleotide polymorphism in the SLC30A8 gene determines ZnT8 autoantibody specificity in type 1 diabetes. Diabetes 57:2693-2697

12. Achenbach $P$, Lampasona V, Landherr U, Koczwara K, Krause S, Grallert H, Winkler C, Pflüger M, Illig T, Bonifacio E, Ziegler AG (2009) Autoantibodies to zinc transporter 8 and SLC30A8 genotype stratify type 1 diabetes risk. Diabetologia 52(9):1881-1888

13. Kawasaki E, Uga M, Nakamura K, Kuriya G, Satoh T, Fujishima K, Ozaki M, Abiru N, Yamasaki H, Wenzlau JM, Davidson HW, Hutton JC, Eguchi K (2008) Association between anti-ZnT8 autoantibody specificities and SLC30A8 Arg325Trp variant in Japanese patients with type 1 diabetes. Diabetologia 51:2299-2302

14. Trabucchi A, Faccinetti NI, Guerra LL, Puchulu FM, Frechtel GD, Poskus E, Valdez SN (2012) Detection and characterization of ZnT8 autoantibodies could help to screen latent autoimmune diabetes in adult-onset patients with type 2 phenotype. Autoimmunity 45(2):137-142

15. Kawasaki E (2012) ZnT8 and type 1 diabetes. Endocr J 59(7):531-537

16. Kawasaki E (2014) Type 1 diabetes and autoimmunity. Clin Pediatr Endocrinol 23(4):99-105

17. Petruzelkova L, Ananieva-Jordanova R, Vcelakova J, Vesely Z, Stechova K, Lebl J, Dusatkova P, Sumnik Z, Coles R, Powell M, Furmaniak J, Rees Smith B, Kolouskova S (2014) The dynamic changes of zinc transporter 8 autoantibodies in Czech children from the onset of Type 1 diabetes mellitus. Diabet Med 31(2):165-171

18. Shivaprasad C, Mittal R, Dharmalingam M, Kumar PK (2014) Zinc transporter- 8 autoantibodies can replace IA-2 autoantibodies 
as a serological marker for juvenile onset type 1 diabetes in India. Indian J Endocrinol Metab 18(3):345-349

19. Chimienti F, Devergnas S, Favier A, Seve M (2004) Identification and cloning of a $\beta$-cell-specific zinc transporter, ZnT8, localized into insulin secretory granules. Diabetes 53:2330-2333

20. Masala S, Cossu D, Palermo M, Sechi LA (2014) Recognition of zinc transporter 8 and MAP3865c homologous epitopes by Hashimoto's thyroiditis subjects from Sardinia: a common target with type 1 diabetes? PLoS One 9(5):e97621

21. Yang L, Luo S, Huang G, Peng J, Li X, Yan X, Lin J, Wenzlau JM, Davidson HW, Hutton JC, Zhou Z (2010) The diagnostic value of zinc transporter 8 autoantibody (ZnT8A) for type 1 diabetes in Chinese. Diabetes Metab Res Rev 26:579-584
22. Salonen KM, Ryhänen S, Härkönen T, Ilonen J, Knip M, Finnish Pediatric Diabetes Register (2013) Autoantibodies against zinc transporter 8 are related to age, metabolic state and HLA DR genotype in children with newly diagnosed type 1 diabetes. Diabetes Metab Res Rev 29(8):646-654

23. Greenbaum CJ, Palmer JP, Kuglin B, Kolb H (1992) Insulin autoantibodies measured by radioimmunoassay methodology are more related to insulin-dependent diabetes mellitus than those measured by enzyme-linked immunosorbent assay: results of the fourth International workshop on the standardization of insulin. J Clin Endocrinol Metab 74:1040-1044 